\title{
Centralisation of treatment and survival rates for cancer
}

\author{
C A STILLER \\ Childhood Cancer Research Group, Department of Paediatrics, University of Oxford
}

SUMmARY Between 1977 and 1984 the proportion of children with malignant disease in Britain initially referred to specialist paediatric oncology centres increased from $44 \%$ to $71 \%$. The percentage varied considerably with type of disease and region of residence. Children with acute non-lymphoblastic leukaemia, non-Hodgkin's lymphoma, Ewing's tumour, rhabdomyosarcoma, and (during 1981-84) osteosarcoma treated at paediatric oncology centres had significantly higher survival rates than those treated elsewhere. Children with cancer should be referred to specialist centres so that they may benefit as early as possible from the latest advances in treatment.

Since the 1960s there has been an increasing trend towards centralisation of the treatment of childhood cancers in Britain. In 1977 the United Kingdom Children's Cancer Study Group (UKCCSG) was formed by a number of consultants specialising in paediatric oncology. Since then there has been a steady increase in membership of the UKCCSG until now nearly every Health Service region in Britain contains a paediatric oncology centre whose senior staff are members of the UKCCSG. This paper describes the increased centralisation of the care of children with cancer since 1977, and compares survival rates for children with some of the principal types of childhood neoplasm who were treated at paediatric oncology centres with those of children treated elsewhere.

\section{Patients and methods}

The Childhood Cancer Research Group (CCRG) maintains the national registry of childhood malignant diseases for Great Britain. The primary source of information is notification to the National Cancer Registration schemes for England, Scotland, and Wales; the CCRG receives a copy of the registration of each patient aged under 15 years.

Since July 1977 the UKCCSG has kept a register of all children with malignant disease who are either under the continuing care of one of its members or whose management is shared with consultants in district general hospitals. The CCRG receives copies of all notifications to this register. By 1984 there were UKCCSG centres in the following cities and towns in Great Britain: Aberdeen, Birmingham, Bristol, Cambridge, Cardiff, Edinburgh, Glasgow, Leeds, Leicester, Liverpool, London (two), Manchester, Newcastle upon Tyne, Nottingham, Sheffield, Southampton, and Sutton. There were also centres in Belfast and Dublin whose patients are not included in the present analyses.

Confirmation of the diagnosis and information on follow up are obtained for children in both registries about five years after diagnosis. The CCRG also routinely receives death certificates for people under the age of 20 dying of neoplasms. Survivors are flagged in the National Health Service Central Registers so that the CCRG is notified of any deaths or embarkations (resulting in loss to follow up). Most survivors in both the CCRG and UKCCSG registries have been followed up at least until the end of June 1986.

Although cancer registration in Britain is population based, it is not quite complete. The proportion of all patients in Britain who are managed at paediatric oncology centres, however, may be estimated as the proportion of the total number of children with a cancer registration who also have a UKCCSG notification.

Comparisons of survival rates between UKCCSG patients and non-UKCCSG patients were made for eight of the principal types of childhood neoplasm: acute non-lymphoblastic leukaemia, Hodgkin's disease, non-Hodgkin's lymphoma, neuroblastoma, Wilms' tumour, osteosarcoma, Ewing's tumour, and rhabdomyosarcoma. All children in these groups were categorised by the type of treatment centre 
responsible for their main treatment: Paediatric oncology centres (this group includes children whose treatment is shared between the main centre and another); other teaching hospitals; and other non-teaching hospitals.

Some hospitals began to participate in the UKCCSG after 1977. For the present analyses, patients at these centres were counted as being treated at other teaching hospitals if diagnosed before consultants at the centre became members of the UKCCSG and as being treated in paediatric oncology centres if diagnosed since their participation in the UKCCSG began.

Actuarial survival rates and the significance of differences between survival curves were calculated using standard methods. ${ }^{1}$ The effect of referral on survival rates for childhood acute lymphoblastic leukaemia is the subject of another study (Stiller and Draper, unpublished observations) and is therefore not considered in detail in the present paper.

\section{Results}

Table 1 shows the estimated percentages of children with cancer in Britain during the period July 1977 to December 1984 who were initially referred to

Table 1 Percentages of children with malignant neoplasms initially referred to paediatric oncology centres 1977-84

\begin{tabular}{|c|c|c|}
\hline \multirow{2}{*}{$\begin{array}{l}\text { Diagnostic group } \\
\text { and age (years) }\end{array}$} & \multicolumn{2}{|c|}{ Years of diagnosis } \\
\hline & $\begin{array}{l}\text { July } \\
1977-1980\end{array}$ & $1981-84$ \\
\hline \multicolumn{3}{|l|}{ Acute lymphoblastic } \\
\hline leukaemia & 64 & 78 \\
\hline \multicolumn{3}{|l|}{ Acute non-lymphoblastic } \\
\hline leukaemia & 62 & 77 \\
\hline Other leukaemia & 49 & 54 \\
\hline Hodgkin's disease & 60 & 67 \\
\hline Non-Hodgkin's lymphoma & 67 & 77 \\
\hline Neuroblastoma & 73 & 86 \\
\hline Wilms' tumour & 69 & 88 \\
\hline Retinoblastoma & 11 & 27 \\
\hline Medulloblastoma & 45 & 56 \\
\hline \multicolumn{3}{|l|}{ Other central nervous } \\
\hline system & 28 & 31 \\
\hline Osteosarcoma & 36 & 52 \\
\hline Ewing's tumour & 56 & 73 \\
\hline Rhabdomyosarcoma & 68 & 90 \\
\hline Malignant germ cell tumour & 53 & 62 \\
\hline Other malignant disease & 43 & 44 \\
\hline \multicolumn{3}{|l|}{ Age: } \\
\hline $0-4$ & 58 & 72 \\
\hline $5-9$ & 58 & 67 \\
\hline $10-14$ & 42 & 53 \\
\hline Total & 53 & 65 \\
\hline
\end{tabular}

paediatric oncology centres. The proportion of such children increased from $44 \%$ in the second half of 1977 to $71 \%$ in 1984 . This increase took place for children of all ages, but throughout the period a smaller proportion of children aged 10-14 (and especially of those aged 12 and over) were referred to a paediatric oncology centre. The referral rates varied considerably between health regions, ranging in England from $85 \%$ for children living in the North West, to $28 \%$ for those living in Oxford. The rates also varied with diagnostic group from over three quarters for the leukaemias, neuroblastoma, Wilms' tumour and rhabdomyosarcoma to slightly over one quarter for central nervous system tumours other than medulloblastoma. A large proportion of children with retinoblastoma have been treated at two London hospitals (Sanders and Draper, unpublished observations), but until recently these patients were not notified to the UKCCSG.

Table 2 shows the total numbers of patients included in the analyses of survival categorised by type of treatment centre. Children who are in the UKCCSG register but for whom a cancer registration has not yet been received are included in these analyses. Children who died before treatment could be given, including some where the diagnosis was made at necropsy, have been excluded.

For many of the non-UKCCSG children diagnosed from 1981 onwards the diagnosis was based solely on the cancer registration and in a few instances may be changed when the five year follow up is completed. Moreover, registration of nonUKCCSG children for 1981-84 is incomplete. For these reasons the analyses have been carried out separately for children diagnosed in 1977-80 and in

Table 2 Total numbers of patients included in survival analyses for each diagnostic group categorised by type of treatment centre

\begin{tabular}{|c|c|c|c|c|}
\hline \multirow[t]{2}{*}{ Diagnostic group } & \multicolumn{3}{|c|}{ Treatment centre } & \multirow[t]{2}{*}{ Total } \\
\hline & $\begin{array}{l}\text { Paediatric } \\
\text { oncology } \\
\text { centre }\end{array}$ & $\begin{array}{l}\text { Other } \\
\text { teaching }\end{array}$ & $\begin{array}{l}\text { Other } \\
\text { non- } \\
\text { teaching }\end{array}$ & \\
\hline \multicolumn{5}{|c|}{ Acute non-lymphoblastic } \\
\hline $\begin{array}{l}\text { Hodgkin's disease } \\
\text { Non-Hodgkin's }\end{array}$ & 291 & 81 & 63 & 435 \\
\hline lymphoma & 385 & 56 & 56 & 497 \\
\hline Neuroblastoma & 417 & 45 & 24 & 486 \\
\hline Wilms' tumour & 397 & 44 & 42 & 483 \\
\hline Osteosarcoma & 108 & 71 & 61 & 240 \\
\hline Ewing's tumour & 158 & 49 & 27 & 234 \\
\hline Rhabdomyosarcoma & 284 & 35 & 32 & 351 \\
\hline Total & 2335 & 435 & 345 & 3115 \\
\hline
\end{tabular}


Table 3 Actuarial three year percentage survival rates for children at each type of treatment centre with results of log rank tests for heterogeneity of survival curves among types of treatment centre allowing for age and year of diagnosis

\begin{tabular}{|c|c|c|c|c|c|c|c|c|}
\hline \multirow[t]{3}{*}{ Diagnostic group } & \multicolumn{6}{|c|}{ Treatment centre and years of diagnosis } & \multirow{3}{*}{$\begin{array}{l}\chi^{2} \\
(2 d f)\end{array}$} & \multirow{3}{*}{$\begin{array}{l}p \\
\text { value }\end{array}$} \\
\hline & \multicolumn{2}{|c|}{$\begin{array}{l}\text { Paediatric oncology } \\
\text { centre }\end{array}$} & \multicolumn{2}{|l|}{$\begin{array}{l}\text { Other } \\
\text { teaching }\end{array}$} & \multicolumn{2}{|c|}{$\begin{array}{l}\text { Other } \\
\text { non-teaching }\end{array}$} & & \\
\hline & $1977-80$ & $1981-84$ & $1977-80$ & $1981-84$ & $1977-80$ & $1981-84$ & & \\
\hline Acute non-lymphoblastic leukaemia & 28 & 32 & 23 & (21) & 9 & (6) & $8 \cdot 29$ & $<0.05$ \\
\hline Hodgkin's disease & 90 & 98 & 91 & 85 & 88 & 100 & 0.69 & NS \\
\hline Non-Hodgkin's lymphoma & 56 & 70 & 32 & $(56)$ & 43 & $(58)$ & $10 \cdot 8$ & $<0 \cdot 01$ \\
\hline Neuroblastoma & 32 & 49 & 52 & (54) & (25) & * & $4 \cdot 09$ & NS \\
\hline Wilms' tumour & 83 & 80 & 81 & (77) & 81 & * & $0 \cdot 31$ & NS \\
\hline Osteosarcoma & 39 & 54 & 32 & 24 & 38 & 37 & $4 \cdot 39$ & NS \\
\hline Ewing's tumour & 46 & 50 & 24 & (33) & (43) & (45) & 8.04 & $<0.05$ \\
\hline Rhabdomyosarcoma & 63 & 63 & 25 & (36) & 46 & & $21 \cdot 0$ & $<0 \cdot 0001$ \\
\hline
\end{tabular}

Figures in parentheses are percentages of fewer than 20 patients; ${ }^{*}=$ fewer than 10 patients in the group.

1981-84. This also enabled trends in survival to be examined, allowing for changes in referral patterns. Because older children were less likely to be treated at paediatric oncology centres, age at diagnosis grouped as $0-11$ and $12-14$ years was allowed for in all the analyses.
Table 3 shows the three year survival for children in each diagnostic group, categorised by year of diagnosis and treatment centre, together with the results of significance tests for differences in the survival curves among types of treatment centre, allowing for the effects of age and year of diagnosis.

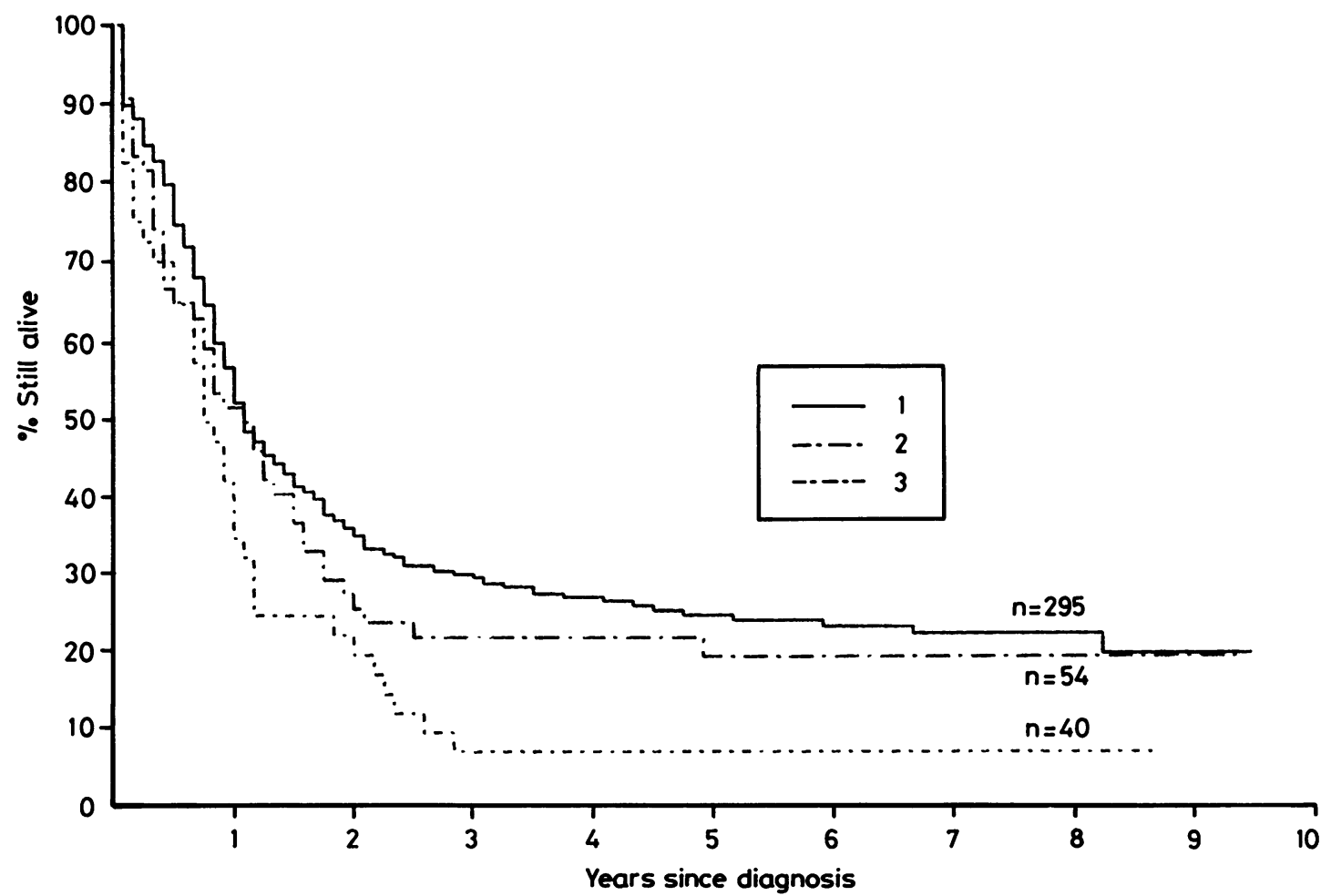

Fig 1 Actuarial survival curves for children with acute non-lymphoblastic leukaemia classified by type of treatment centre. $1=$ Paediatric oncology centres; 2 =other teaching hospitals; $3=$ other hospitals. 
Fig 1 shows the significant variation among types of treatment centre in the survival for children with acute non-lymphoblastic leukaemia. This difference held for both age groups and in both calendar periods and was largely accounted for by the low survival among children in the non-teaching hospitals.

The survival for children with Hodgkin's disease was high with over $90 \%$ expected to survive at least three years, and there was no evidence of variation among types of treatment centre. Between 1977-80 and 1981-84 there was evidence of further improvement in the already high survival rate.

There was a highly significant difference in survival in non-Hodgkin's lymphoma among the types of treatment centre, with the highest rates being seen at paediatric oncology centres. This difference held for both age groups. There was a marked increase in survival between 1977-80 and 1981-84 among children treated at paediatric oncology centres and elsewhere, but the rates for other hospitals during 1981-84 only reached those attained at the paediatric oncology centres during 1977-80 (fig 2).

Analysis of the survival for neuroblastoma showed no clear heterogeneity among types of treatment centre. Tumour stage according to the Evans system ${ }^{2}$ was given for most of the patients, however, and the paediatric oncology centres had a considerably higher proportion of stage III and IV patients (who have a worse prognosis) than other hospitals. At paediatric oncology centres there was a substantial improvement in survival rates between 1977-80 and 1981-84. Three year survival at these centres increased from $32 \%$ to $49 \%$ between the two calendar periods for all stages combined, from $33 \%$ to $61 \%$ for stage III, and from $11 \%$ to $21 \%$ for stage IV.

There was no difference in survival for Wilms' tumour among types of treatment centre even allowing for the fact that children with metastatic disease at diagnosis appeared to be more likely to be referred to a paediatric oncology centre, nor was there any change between $1977-80$ and $1981-84$.

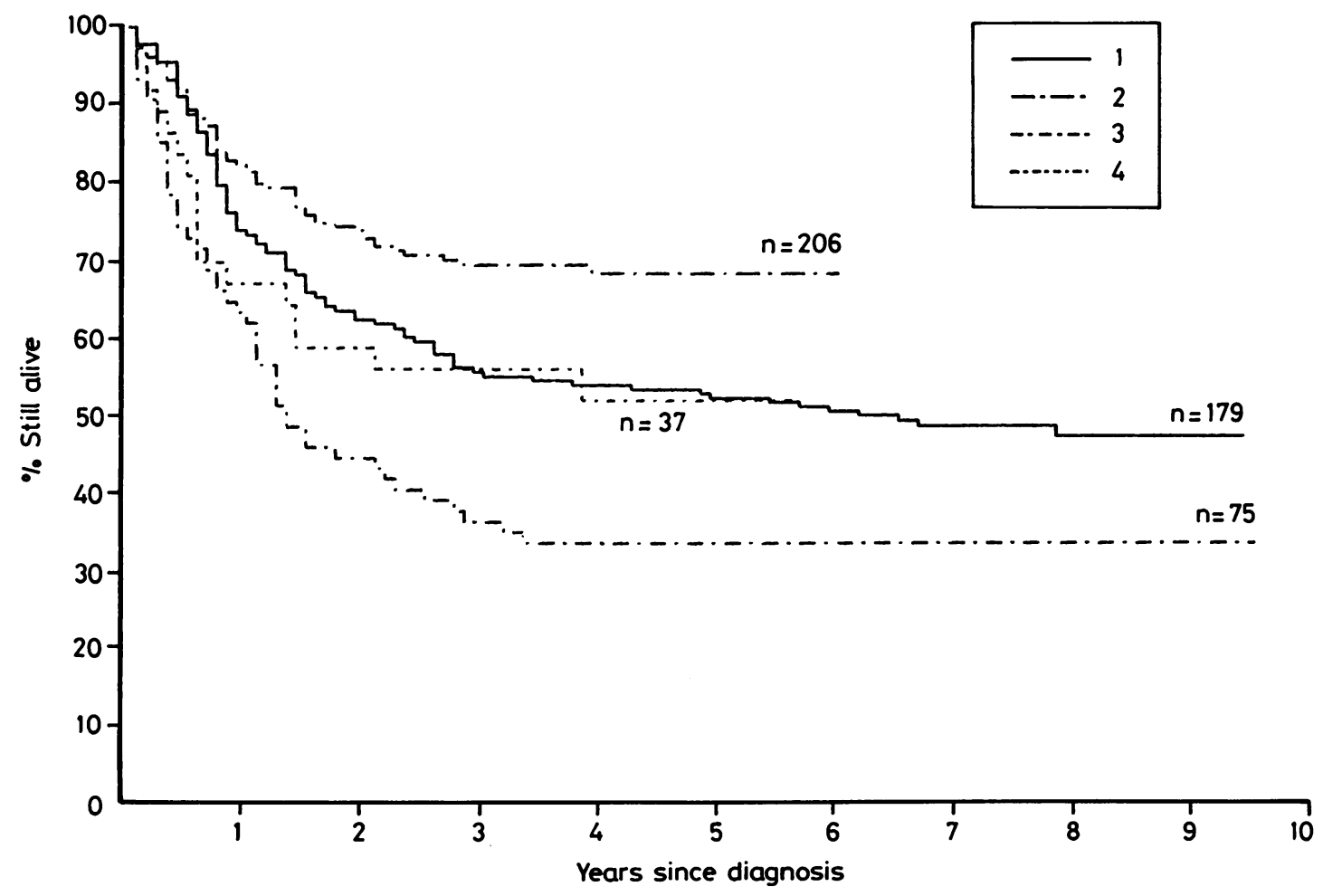

Fig 2 Actuarial survival curves for children with non-Hodgkin's lymphoma classified by type of treatment centre. I=Paediatric oncology centres, 1977-80; 2=paediatric oncology centres, 1981-4; 3=all other hospitals, 1977-80; $4=$ all other hospitals, 1981-4. 
During 1977-80 survival for childhood osteosarcoma was similar in all types of treatment centre, with rates of about $36 \%$ at three years and slightly under $30 \%$ at five years. For 1981-84, there was a significant variation, with children treated at paediatric oncology centres having a considerably higher probability of survival. The survival for the children at these centres in this period showed a definite increase over earlier years (fig 3); there was little evidence of a corresponding improvement elsewhere.

There were significant differences among types of treatment centre in survival for Ewing's tumour, with a greater proportion of children surviving at paediatric oncology centres. The prognosis for Ewing's tumour varies with primary site, being best for tumours in the long bones of the limbs and worst for those in the ribs and pelvis. In the analysis of survival by site of tumour there was a significant variation among types of treatment centre for patients with primaries in both of these sites and in the miscellaneous other sites-in each case with a higher survival rate at the paediatric oncology centres. These centres treated proportionally more patients with prognostically unfavourable primary sites. There was no evidence of any substantial improvement in overall survival between 1977-80 and 1981-84.

There was a significant variation in the survival for rhabdomyosarcoma, again with a higher proportion of survivors at paediatric oncology centres (fig 4). This was true for both age groups and both calendar periods. There was no evidence of an increase in survival rates between 1977-80 and 1981-84.

\section{Discussion}

The benefits of referral to specialist treatment hospitals for patients with cancer have been widely discussed. ${ }^{34}$ For children there has until recently been little published about the advantages of treatment in specialist centres, but in the past decade four papers have reported beneficial effects on

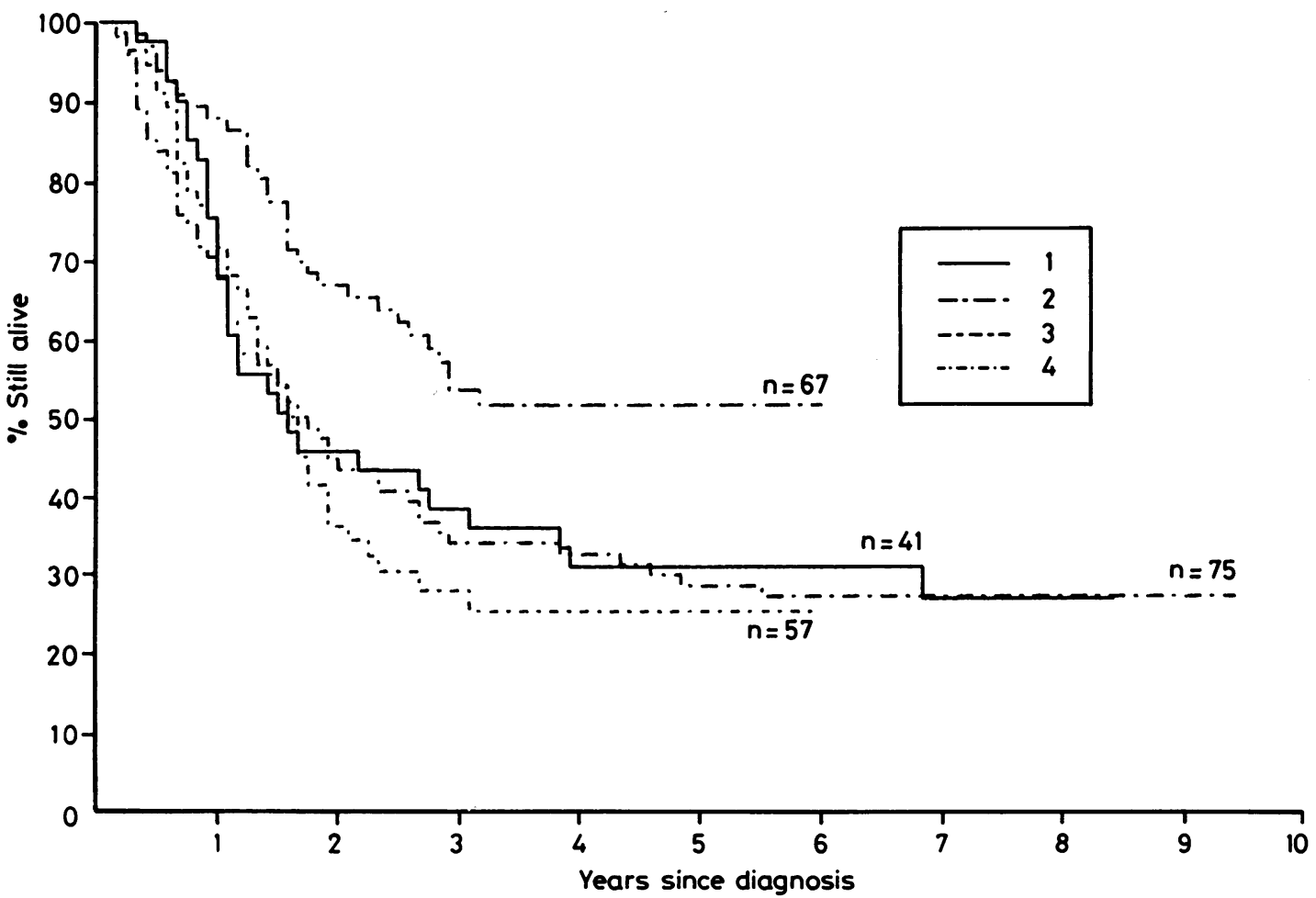

Fig 3 Actuarial survival curves for children with osteosarcoma classified by type of treatment centre. 1=Paediatric oncology centres, 1977-80; $2=$ paediatric oncology centres, 1981-4; 3=all other hospitals, 1977-80; 4=all other hospitals, 1981-4. 


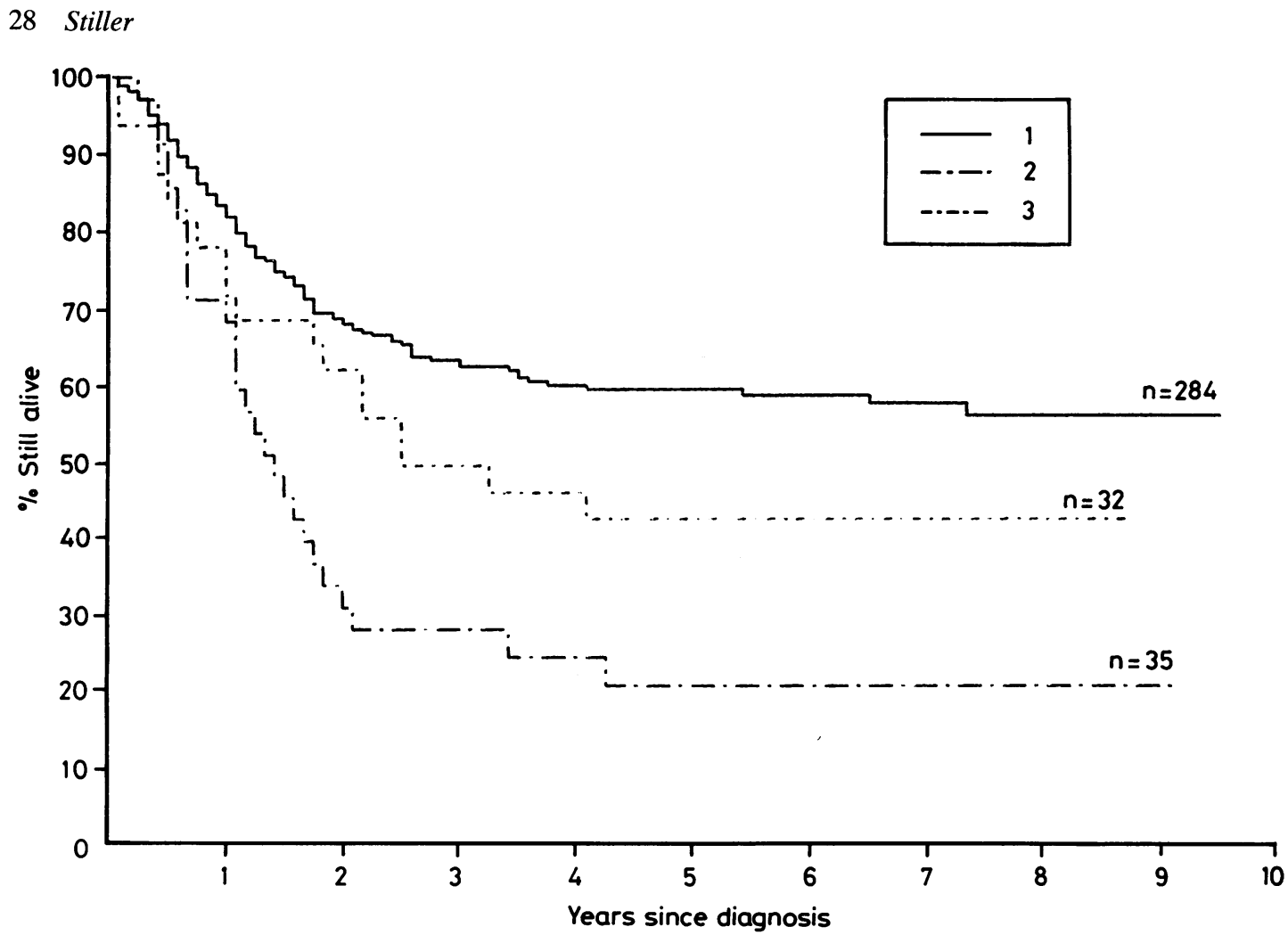

Fig 4 Actuarial survival curves for children with rhabdomyosarcoma classified by type of treatment centre. $1=$ Paediatric oncology centres; 2 =other teaching hospitals; $3=$ other hospitals.

survival when treatment was given according to standardised protocols for children with Wilms' tumour, ${ }^{56}$ acute lymphoblastic leukaemia, ${ }^{7}$ and medulloblastoma. ${ }^{8}$ Kramer et al examined the effect of place of treatment on the survival for children with Wilms' tumour, rhabdomyosarcoma, and medulloblastoma in the population based Greater Delaware Valley Pediatric Tumor Registry. ${ }^{9}$ A recent analysis of data about acute lymphoblastic leukaemia in Britain during the 1970s (Stiller and Draper, unpublished observations) showed that survival was higher among children who were included in nationally organised trials or referred to specialist centres treating large numbers of patients.

In Britain there has been well established national cooperation in trials of treatment for acute lymphoblastic leukaemia, coordinated by the Medical Research Council since the 1960 s. ${ }^{10}$ The Council also supported trials in the treatment of Wilms' tumour, ${ }^{11}$ and a few of the largest children's hospitals took part in some of the studies of other childhood cancers organised by the International
Society of Paediatric Oncology, but in general there was no formal basis in Britain for national collaborative studies of the treatment of childhood cancer until the formation of the UKCCSG. The data presented here show that since 1977 there has been a definite trend towards treatment of children with malignant disease in paediatric oncology centres, with UKCCSG members now being responsible for at least two thirds of newly diagnosed patients.

The survival analysis showed a much lower survival for patients with acute non-lymphoblastic leukaemia treated at non-teaching hospitals, possibly because they would have had little experience in treating children with this disease. Although the prognosis is still poor, the three year survival of about $26 \%$ during $1977-84$ was considerably higher than the $10 \%$ for $1971-74 .{ }^{12}$ This improvement is largely attributable to the adoption of more effective chemotherapy, though bone marrow transplantation may have also increased the number of survivors.

By the early 1970s Hodgkin's disease was already one of the childhood cancers with the best prognosis, with a three year survival of over $80 \% .^{12}$ 
Since then there has been further steady improvement, and nowadays well over $90 \%$ of patients survive for at least three years. These improvements are similar to those achieved in adults during the same period. ${ }^{13}$

There has been a considerable improvement in survival for childhood non-Hodgkin's lymphomas since 1971-74 when the three year survival was only $28 \%$ compared with over $65 \%$ for patients diagnosed in the $1980 \mathrm{~s}^{12}$ The adoption of more intensive multiple drug regimens is probably responsible for the greatly improved outlook. ${ }^{14} 15$

Although survival rates for neuroblastoma are still low in comparison with many other childhood cancers, the prospects are much better than in the early 1970 s when long term survival was only $15 \% .{ }^{12}$ The survival was lower at paediatric oncology centres than elsewhere, maybe because these centres were seeing a much larger proportion of patients with advanced disease. The greater number of patients with stage III and IV tumours at these centres could be an artefact caused by more careful staging than at other hospitals, but this is unlikely as the proportions of patients in these stages at the paediatric oncology centres remained constant between the two calendar periods (at around 19\% for stage III and $54 \%$ for stage IV), and most of the survivors at other hospitals had localised tumours which were apparently cured without chemotherapy. During the past decade there have been considerable improvements in the treatment of advanced neuroblastomas at specialist centres with encouraging results; more patients have been treated with chemotherapy regimens that include cisplatin and melphalan. ${ }^{16}$

Survival rates everywhere were high for Wilms' tumour compared with that of earlier years. Previous reports showed an advantage in survival for patients treated in clinical trials or at specialist centres. ${ }^{56}$ There was no significant difference in survival in the present series between children managed at paediatric oncology centres and those managed elsewhere. Although some of the children in other hospitals have been included in the UKCCSG study and treated using that protocol, however, it seems that others were given more radiotherapy or chemotherapy than was appropriate for their stage and histological subtype within the study. Kramer et al also found no significant differences in survival between cancer centres and other hospitals. ${ }^{9}$ They suggested that this might be because by the time of their study Wilms' tumour was readily curable by regimens that had been published and were widely used.

Survival rates for childhood osteosarcoma in 1977-80 showed a modest improvement over
1971-74, possibly because most of the patients diagnosed in the more recent period received adjuvant chemotherapy. There was a much greater improvement for 1981-84 but this was limited to children managed at paediatric oncology centres. Detailed information is not yet available, but more effective chemotherapy might have been given at these centres while other hospitals were continuing with earlier regimens.

Overall survival for Ewing's tumour showed no change during 1977-84, and indeed was no higher than for patients diagnosed during 1971-74 (51\% at three years). ${ }^{12}$ There was, however, a highly significant variation among types of treatment centre in favour of the paediatric oncology centres. Children treated at other hospitals usually received both radiotherapy and chemotherapy in similar combinations to those used at paediatric oncology centres.

The survival rate for rhabdomyosarcoma showed a definite improvement over $1971-74$ (28\% at three years) ${ }^{12}$ but there was little evidence of further advances within the period under review. Again the survival rates for the paediatric oncology centres were superior to those elsewhere, a result similar to that reported by Kramer et al in the United States. ${ }^{9}$ There were a few patients at other hospitals who initially had radiotherapy but no chemotherapy, but most appeared to have received standard combinations of cytotoxic drugs.

Details about doses and duration of chemotherapy were not generally available in the registry. The poor survival rates at other hospitals for Ewing's tumour and rhabdomyosarcoma, which are often treated using similar combinations of chemotherapy, however, suggest that although appropriate drugs were used they may not always have been given in adequate doses.

Analyses of survival rates based on registration data are susceptible to various sorts of bias. In removing the untreated patients and stratifying the rest by age, year of diagnosis, and where appropriate primary site, however, the most important confounding factors should have been allowed for in comparing the rates for patients referred to different types of treatment centre.

For certain diagnostic groups, notably acute nonlymphoblastic leukaemia and non-Hodgkin's lymphoma, there was a considerably higher initial mortality at the non-specialist centres, suggesting failure to achieve remission. Despite the exclusion of untreated patients from the survival analyses, a few patients might have been diagnosed too late for referral to a paediatric oncology centre but nevertheless received some treatment at other hospitals. This is unlikely to account for the difference 
between types of treatment centre, however, because when the analyses were repeated with all children who survived under one month excluded, the significance level of the difference in survival did not change for any diagnostic group.

There have been considerable improvements in recent years in the survival for several types of childhood cancer, presumably resulting from advances in treatment, and the results obtained at specialist paediatric oncology centres have tended to be better than those achieved elsewhere. The results presented here underline the importance of referring patients to specialist centres where they may benefit as early as possible from the latest advances in treatment. In some instances children receive less aggressive treatment than previously, still with a high probability of survival but with a reduced risk of long term sequelae. Centralisation of treatment may result in increased cost to the family ${ }^{17}$ and a greater workload at the paediatric oncology centres, but these disadvantages can be alleviated by closer cooperation between specialist centres and local hospitals, especially during maintenance therapy.

I thank the consultants and general practitioners who provided the information on which this paper is based. I am grateful to the Office of Population Censuses and Surveys, the Information Services Division of the Common Services Agency of the Scottish Health Service, the General Register Office for Scotland, regional cancer registries and the UKCCSG for providing copies of notifications of childhood cancer cases. I thank the National Health Service Central Registers for notification of deaths and the flagging of survivors. I am grateful to Mrs EM Roberts for her part in collecting the medical records and secretarial help, to $\mathrm{Mr} \mathrm{H}$ Fearnley and Dr M Potok for extensive computing work, and to Dr LM Kinnier Wilson for providing data from the Oxford Survey of Childhood Cancers. I am indebted to many colleagues for valuable discussions of earlier drafts, especially Dr GJ Draper, Dr P Morris Jones and Dr MG Mott.

The CCRG is supported by the Department of Health and Social Security, the Scottish Home and Health Department, the Cancer Research Campaign and the Leukaemia Research Fund. Collection of data was also supported by the Marie Curie Memorial Foundation.

\section{References}

${ }^{1}$ Peto R, Pike MC, Armitage P, et al. Design and analysis of randomised clinical trials requiring prolonged observation of each patient: II. Analysis and examples. Br J Cancer 1977;35: 1-39.

2 Evans AE, D'Angio GJ, Randolph J. A proposed staging for children with neuroblastoma. Cancer 1971;27:374-8.

${ }^{3}$ Bagshawe KD, Begent RHJ, Newlands ES, Rustin GJS. What sort of oncology team should treat testicular teratoma? Lancet $1985 ; \mathbf{i}: 930$.

4 Oliver RTD. Rare cancers and specialist centres. Br Med J 1986;292:641-2.

5 Griffel M. Wilms' tumor in New York State: epidemiology and survivorship. Cancer 1977;40:3140-5.

${ }^{6}$ Lennox EL, Stiller CA, Morris Jones P, Kinnier Wilson LM. Nephroblastoma: treatment during 1970-73 and the effect on survival of inclusion in the first MRC trial. $\mathrm{Br}$ Med $J$ 1979;2:567-9.

7 Meadows AT, Kramer S, Hopson R, et al. Survival in childhood acute lymphocytic leukaemia: effect of protocol and place of treatment. Cancer Investigation 1983;1:49-55.

${ }^{8}$ Duffner K, Cohen ME, Flannery JT. Referral patterns of childhood brain tumours in the State of Connecticut. Cancer 1982;50:1636-40.

${ }^{9}$ Kramer S, Meadows AT, Pastore G, et al. Influence of place of treatment on diagnosis, treatment and survival in three pediatric solid tumors. J Clin Oncol 1984;2:917-23.

${ }^{10}$ Medical Research Council Working Party on leukaemia in childhood. Improvement in treatment for children with acute lymphoblastic leukaemia. Lancet 1986;i:408-11.

11 Medical Research Council Working Party on embryonal tumours in childhood. Management of nephroblastoma in childhood: clinical study for two forms of maintenance chemotherapy. Arch Dis Child 1978;53:112-19.

12 Draper GJ, Birch JM, Bithell JF, et al. Childhood cancer in Britain: incidence, survival and mortality. Studies on medical and population subjects 37. London: HMSO, 1982.

${ }^{13}$ Kaplan H. Hodgkin's disease, 2nd ed. Cambridge: Harvard University Press, 1980.

${ }^{14}$ Mott MG, Chessells JM, Willoughby ML, et al. Adjuvant low dose radiation in childhood $\mathrm{T}$ cell lymphoma/leukaemia. $\mathrm{Br} \mathrm{J}$ Cancer 1984;50:457-62.

${ }^{15}$ Mott MG, Eden OB, Palmer MK. Adjuvant low dose radiation in childhood non-Hodgkin's lymphoma. $\mathrm{Br} J$ Cancer 1984;50:463-9.

${ }^{16}$ Pritchard J, McElwain TJ, Graham-Pole J. High dose melphalan with autologous marrow for treatment of advanced neuroblastoma. Br J Cancer 1982;45:86-94.

17 Bodkin CM, Pigott TJ, Mann JR. Financial burden of childhood cancer. Br Med J 1982;284:1542-4.

Correspondence to Mr CA Stiller, Childhood Cancer Research Group, Radcliffe Infirmary, Oxford OX2 6HE.

Received 15 May 1987 